\title{
SHORT REPORT \\ Impact of an influenza vaccine educational programme on healthcare personnel
}

\author{
R. RODRÍGUEZ-FERNÁNDEZ ${ }^{1,2 *}$, A. B. MARTÍNEZ-LÓPEZ ${ }^{1}$, \\ J. PÉREZ-MORENO ${ }^{1}$, M. I. GONZÁLEZ-SÁNCHEZ ${ }^{1,2}$, \\ F. GONZÁLEZ-MARTÍNEZ ${ }^{1,2}$, T. HERNÁNDEZ-SAMPELAYO ${ }^{2,3}$ AND \\ A. MEJIAS ${ }^{4}$ \\ ${ }^{1}$ Section of General Paediatrics, Hospital Gregorio Marañón, Madrid, Spain \\ ${ }^{2}$ Gregorio Marañón Health Research Institute, Madrid, Spain \\ ${ }^{3}$ Section of Infectious Diseases, Department of Paediatrics, Hospital Gregorio Marañón, Madrid, Spain \\ ${ }^{4}$ Division of Paediatric Infectious Diseases, Centre for Vaccines and Immunity, Nationwide Children's Hospital \\ and The Ohio State University College of Medicine, Columbus, OH, USA
}

Received 19 August 2015; Final revision 18 March 2016; Accepted 21 March 2016; first published online 7 April 2016

\section{SUMMARY}

Influenza vaccination has been shown to be the most effective preventive strategy to reduce influenza-related morbidity and mortality in high-risk groups. Despite healthcare personnel (HCP) being considered part of such high-risk groups, their vaccination coverage is low in Europe. In January 2012, we distributed an 18-question survey regarding influenza vaccination to HCP at Gregorio Marañon Paediatric Hospital, in Madrid, Spain. After we documented that only $\sim 30 \%$ of HCP were vaccinated an educational programme was implemented in October 2012 before the next influenza season. In January 2013, the same survey delivered again to all HCP documented a significant increase in vaccination rates (from $30 \%$ to $40 \%, P=0 \cdot 007$ ) mainly among physicians and for patients' protection. In summary we found that a simple and inexpensive educational programme significantly improved the uptake of influenza vaccination in HCP in our centre. Nevertheless, vaccination rates remained low, and broader and updated campaigns are needed to overcome perception barriers.

Key words: Educational programme, healthcare personnel, influenza vaccination.

Influenza can lead to severe disease and death in populations at high risk. In the United States 5-20\% of the whole population suffer from influenza each year. In Spain rates of hospitalization for severe confirmed influenza were $\sim 5.76$ cases $/ 100000$ during the 2010-2011 season [1-3].

\footnotetext{
* Author for correspondence: Dr R. Rodríguez-Fernández, Sección of General Paediatrics, Hospital Infantil Gregorio Marañón, Calle O’Donnell 50, 28007, Madrid, Spain.

(Email: rrodriguezf.hgugm@salud.madrid.org)
}

Healthcare personnel (HCP) are considered one of the high-risk categories because of their frequent exposure to patients with influenza, and also because they can serve as potential vectors of transmission. However, influenza vaccine coverage in this group has been reported to be as low as $25 \%$ in adult hospitals in Spain compared to 30\% in Europe and up 77\% in the United States [2-5].

The present study was designed to determine whether implementation of an influenza vaccine educational programme at a three-level paediatric hospital improved vaccination uptake in HCP and the 
reasoning behind those changes. To this end, we conducted a prospective, cross-sectional study at Hospital Infantil Gregorio Marañón, Madrid, Spain. This hospital, which is part of Gregorio Marañón Medical Centre, has $\sim 700$ employees and provides coverage for a population of 750000 inhabitants, including 95000 children. Of those, each year $\sim 6800$ patients aged $<16$ years receive medical care at the hospital.

The Paediatric Hospitalist and Infectious Diseases Sections designed an epidemiological survey, which was distributed to all HCP before and after a vaccine educational programme was implemented in October 2012. The same survey was distributed in January 2012 (pre-programme) and in January 2013 (posteducational programme) after the influenza vaccination campaign ended for the 2012 and 2013 seasons, respectively. The survey assessed whether HCP received influenza vaccine, but also investigated potential problems or barriers to vaccination (see Supplementary Appendix). It was composed of 18 open questions, allowing one or more possible answers including: demographic information (age, gender), professional activity (degree, work unit, shift work) and information about influenza vaccination: vaccination in previous seasons, location and month of vaccination during the current season, side-effects associated with the vaccine if any, reasons for accepting or declining vaccination, as well as suggestions to increase vaccination coverage. All responses were captured in an anonymous fashion and data was de-identified.

The vaccine educational programme was led by three attending physicians as part of the Hospitalist group and sessions were provided from September to October 2012, between two influenza seasons. The programme was not mandatory and included an interactive session that provided information about the virus and infection, the risk of nosocomial transmission, and the effectiveness of influenza vaccination. It was offered to all paediatric hospital HCP including: physicians, registered nurses, radiologists, laboratory personnel, and also administrative staff and managers and it was attended only once. Sessions were held at each and all paediatric ward conference rooms. For attending physicians and medical residents two sessions were offered, one in September and another in October as part of the paediatric lectures scheduled for that month. For the remaining HCP (registered nurses, clinical assistants, and other non-medical personnel) a total of eight sessions were scheduled to cover all shifts (four in the morning, two in the afternoon and two in the evening). The programme did not offer influenza vaccination; however, it facilitated access to the vaccine by providing the specific time and location for influenza immunization at the Epidemiology and Occupational Health Unit at Gregorio Marañón General Hospital. Two types of inactivated influenza vaccine were offered during the study period: the trivalent virome adjuvanted subunit influenza vaccine (Inflexal V; Crucell, UK) and the trivalent non-adjuvanted influenza vaccine (Fluarix; GSK, USA).

Descriptive analyses were performed using frequency distributions and rates. Means ( \pm s.D.) or medians (25th75th percentiles) were used to summarize patients' demographic and baseline characteristics. Groups were compared using Student's $t$ test or Mann-Whitney test for continuous variables. $\chi^{2}$ or Fisher's exact tests were used for associations between categorical variables as appropriate. A two-tailed $P$ value $<0.05$ was considered significant. All statistical analysis were performed using SPSS v. 18.0 software (SPSS Inc., USA).

Surveys were distributed to 705 active employees during the first period (P1, January 2012) and to 687 employees during the second period ( $\mathrm{P} 2$, January 2013) with similar response rates: P1 $(55 \cdot 6 \%, n=392)$ and P2 $(55 \cdot 7 \%, n=383)$. While participation of physicians, medical residents and medical students in the survey significantly increased from $\mathrm{P} 1$ to $\mathrm{P} 2$, participation of registered nurses and clinical assistants decreased (Supplementary Table S1). Nevertheless, during both periods, HCP documented that the most effective measures to help increase influenza vaccination would be information about the vaccine and disease, and facilitating access to vaccination.

During these two periods the rates of influenza vaccination in Gregorio Marañón Medical Centre (the combined adult and paediatric hospital) were low, $22.5 \%$ in $\mathrm{P} 1$ and $23.8 \%$ in $\mathrm{P} 2$. On the other hand rates of vaccination exclusively in the paediatric hospital increased after the educational programme was implemented from $30 \cdot 4 \%$ in $\mathrm{P} 1$ vs. $39 \cdot 7 \%$ in $\mathrm{P} 2(P=$ 0.007). Forty-five percent of those who participated in the programme received the influenza vaccine compared to $21 \%$ of those who did not participate $(P=0 \cdot 01)$. Information according to vaccine administration during both periods is given in Supplementary Table S2. The programme was attended by $210 \mathrm{HCP}$ ( 80 physicians, 64 registered nurses, 48 clinical assistants and $18 \mathrm{HCP}$ included in other categories). Of those vaccinated after the programme's implementation, $62 \%$ received information about influenza immunization in the hospital through the campaign while the proportion of those that also received that information and 
Table 1. Characteristics of healthcare personnel (HCP) included in the study before and after implementation of the influenza vaccine educational programme

\begin{tabular}{llll}
\hline \hline & $\begin{array}{l}\text { P1 (January 2012) } \\
n / N(\%)\end{array}$ & $\begin{array}{l}\text { P2 (January 2013) } \\
n / N(\%)\end{array}$ & $P$ value \\
\hline Gender (\% females) & $344 / 392(88)$ & $319 / 383(83 \cdot 4)$ & $0 \cdot 117$ \\
Age (years) & & & $0 \cdot 393$ \\
$20-30$ & $83 / 392(21 \cdot 2)$ & $82 / 383(21 \cdot 5)$ & \\
$31-40$ & $77 / 392(19 \cdot 6)$ & $86 / 383(22 \cdot 5)$ & \\
$41-50$ & $121 / 392(30 \cdot 9)$ & $97 / 383(25 \cdot 4)$ & \\
$51-60$ & $93 / 392(23 \cdot 7)$ & $96 / 383(25 \cdot 2)$ & \\
$\geqslant 61$ & $18 / 392(4 \cdot 6)$ & $20 / 383(5 \cdot 4)$ & \\
Rates of influenza vaccination & $119 / 392(30 \cdot 4)$ & $152 / 383(39 \cdot 7)$ & $0 \cdot 007$ \\
Vaccination coverage groups & & & \\
$\quad$ Clinical assistants & $22 / 119(18 \cdot 5)$ & $22 / 81(27 \cdot 2)$ & $0 \cdot 175$ \\
$\quad$ Registered nurses & $53 / 168(31 \cdot 5)$ & $39 / 126(31)$ & $1 \cdot 000$ \\
$\quad$ Medical residents* & $6 / 22(27 \cdot 3)$ & $18 / 42(42 \cdot 9)$ & $0 \cdot 025$ \\
$\quad$ Attending physicians & $24 / 43(55 \cdot 8)$ & $58 / 83(69 \cdot 9)$ & $0 \cdot 057$ \\
Vaccination rates in HCP assigned to high-risk units & $47 / 170(27 \cdot 6)$ & $45 / 91(49 \cdot 5)$ & $0 \cdot 001$ \\
Reasons for vaccination & & & \\
$\quad$ Self-protection & $102 / 119(86)$ & $106 / 150(71)$ & $0 \cdot 005$ \\
$\quad$ Patients' protection & $70 / 119(58)$ & $106 / 150(71)$ & $0 \cdot 058$ \\
\hline \hline
\end{tabular}

Data were compared using Student's $t$ test or Mann-Whitney test for continuous variables and $\chi^{2}$ or Fisher's exact tests for associations between categorical variables as appropriate.

* The majority of medical residents were paediatric residents.

declined vaccination was lower $(49 \%, P=0 \cdot 01)$. The main reasons for declining vaccination were concerns about vaccine efficacy (P1: $35 \%$ vs. P2: 32\%), sideeffects (P1: $18 \%$ vs. P2: $19 \cdot 5 \%$ ) and lack of access to the vaccine (P1: $14 \%$ vs. $\mathrm{P} 2: 13 \cdot 8 \%)$. On the other hand, the main reasons that prompted influenza vaccination in HCP changed during the study period. During P1 HCP received the vaccine mainly for self-protection and then for patients' protection. However, during P2 patients' protection became as important as selfprotection (Table 1). Overall 15\% of HCP reported mild vaccine side-effects including pain, fever or discomfort at the injection site.

Physicians were the HCP group with the highest rate of vaccination in $\mathrm{P} 1$ and $\mathrm{P} 2(>55 \%)$; however, the overall rates of influenza vaccination did not change significantly according to the different HCP groups except for medical residents (most of them paediatric residents) whose rates of vaccination increased during P2 $(P=$ $0 \cdot 025$, Table 1$)$. Males received influenza vaccine more commonly than females during P1 (50.8\%vs. $27 \cdot 7 \%$, $P=0.001)$ with a trend towards higher vaccination during $\mathrm{P} 2(50 \cdot 8 \%$ vs. $37 \cdot 6 \%, P=0 \cdot 08)$. HCP on the afternoon shifts were vaccinated less frequently in both periods (P1: $12 \cdot 5 \%$; $\mathrm{P} 2: 17 \cdot 6 \%$ ) than those working during morning shifts (P1: 34.2\%; P2: 48.3\%) and night shifts (P1: $36 \cdot 7 \%$; $\mathrm{P} 2: 37 \cdot 5 \%)$ and this difference was statistically significant $(P=0 \cdot 001)$. Numbers of HCP assigned to high-risk units (haematology/oncology, neonatal intensive care unit, paediatric intensive care unit, and other units caring for immunocompromised patients) were similar between P1 and P2 [43.3\% (170/ 392) vs. $34 \cdot 7 \%$ (133/383), respectively, $P=0 \cdot 091]$. Nevertheless, rates of vaccination significantly increased from $27 \cdot 6 \%$ to $49 \cdot 5 \%$ in this high-risk HCP group after the educational programme was implemented (Table 1).

The main objectives of this study were first to determine the baseline rates of influenza vaccination in HCP at our institution, to implement an influenza vaccine educational programme, and to evaluate the effectiveness of influenza vaccine uptake in HCP after the implementation of the programme. We observed that the percentage of influenza vaccination in HCP at our hospital was low $(\sim 30 \%)$, but we also demonstrated that with a proper educational programme and provision of information about the disease and risk factors, vaccination rates increased.

Despite that HCP knowledge and awareness of the importance of immunization should be higher than that for the general population, we observed a disappointingly low percentage of vaccinated HCP in our study, which is similar to that reported in other European studies but lower than that reported in US-based studies $[1,6]$. The refusal of HCP to be 
vaccinated has been observed before with other transmissible diseases. As an example, vaccination against hepatitis B did not increase until the consequences of long-term carriage and development of chronic disease was realized. The reasons for better vaccine uptake in United States-based vs. European-based studies may be related to several factors including educational programmes, easy access to vaccines free of charge, the use of declination forms and the fact that an increasing number of healthcare organizations in the United States are implementing mandatory immunization policies. In fact, studies have shown that the main measure that has effectively increased vaccine uptake between HCP is the implementation of institutional policies where vaccination is not optional but rather mandatory in order to guarantee patients' protection. In agreement with other studies that have shown the effectiveness of influenza immunization campaigns to promote an increase in vaccination uptake, we documented a significant increase in the number of vaccinated HCPs, mainly physicians, during P2 after the educational programme was implemented [7-10].

A poorly protective vaccine due to major antigenic changes could have been a concern, as recently shown by the low 2014/2015 vaccine effectiveness (VE) against influenza $\mathrm{A}(\mathrm{H} 3 \mathrm{~N} 2)(18-23 \% \mathrm{VE})$ and $\mathrm{B}$ $(45 \% \mathrm{VE})$ viruses. In addition, studies conducted in different parts of the world have shown that the reasons for HCP declining influenza immunization are mostly related to the perception that the illness is mild or not severe enough, and also to concerns regarding adverse events, needles, and the lack of contact with high-risk patients, which was also documented in our study $[1,7,8,10]$. Overall it appears that the main reasons for opting out of influenza vaccination are similar regardless of the study location. Nevertheless, we believe that with proper education via immunization campaigns these perceptions can be modified. In fact, our low-cost educational intervention showed a significant increase in the percentage of overall vaccination in HCP, mainly in medical personnel caring for high-risk patients. We also observed that the main reasons for HCP being vaccinated changed after the implementation of the educational programme, with patients' protection being the main factor favouring vaccination. We believe that this is a major conceptual change that also reflected the effectiveness of the educational intervention $[1,6]$.

Our study has limitations. There may be a selection bias since it is possible that the responses to the survey were driven by HCP that were vaccinated. Nevertheless, a similar proportion $(\sim 56 \%)$ of HCP responded to the survey before and after the implementation of the educational programme. In addition, $60 \%$ of responders acknowledged that they did not receive the influenza vaccine compared to $40 \%$ that did, which suggest that with limitations the survey captured the rates of vaccination. Last, although the comparison between the preand post-programme periods lacks a control group, the methodology used to distribute the survey and gather the data was similar during both study periods, which makes the analyses comparable.

In summary and considering the limitations, we showed that a simple and inexpensive health educational programme significantly improved the uptake of influenza vaccination in HCP, mainly in physicians, in our centre. Nevertheless, vaccination rates remained low, and broader and updated campaigns are needed to overcome perception barriers in order for influenza vaccine to be optimally administered in the healthcare setting.

\section{SUPPLEMENTARY MATERIAL}

For supplementary material accompanying this paper visit http://dx.doi.org/10.1017/S0950268816000716.

\section{ACKNOWLEDGEMENTS}

The authors thank all the staff at the Children's Hospital, the Occupational Health Service and Human Resources of Gregorio Marañón Hospital.

\section{DECLARATION OF INTEREST}

None.

\section{REFERENCES}

1. Llupia A, et al. Vaccination behaviour influences selfreport of influenza vaccination status: a cross-sectional study among health care workers. PLoS ONE 2012; 7: e39496.

2. Black CL, et al. Influenza Vaccination coverage among health care personnel-United States, 2014-15 influenza season. Morbidity and Mortality Weekly Report 2015; 64: 993-999.

3. de Juanes JR, et al. Influenza vaccination coverage among hospital personnel over three consecutive vaccination campaigns (2001-2002 to 2003-2004). Vaccine 2007; 25: 201-204.

4. Dolan GP, et al. Vaccination of health care workers to protect patients at increased risk for acute respiratory disease. Emerging Infectious Diseases 2012; 18: 1225 1234. 
5. Poland GA, et al. Receptivity to mandatory influenza vaccination policies for healthcare workers among registered nurses working on inpatient units. Infection Control and Hospital Epidemiology 2008; 29: 170-173.

6. Dominguez A, et al. Knowledge of and attitudes to influenza vaccination in healthy primary healthcare workers in Spain, 2011-2012. PLoS ONE 2013; 8: e81200.

7. Llupia A, et al. New interventions to increase influenza vaccination rates in health care workers. American Journal of Infection Control 2010; 38: 476-481.
8. Llupia A, et al. Evaluating influenza vaccination campaigns beyond coverage: a before-after study among health care workers. American Journal of Infection Control 2013; 41: 674-678.

9. Looijmans-van den Akker I, et al. How to develop a program to increase influenza vaccine uptake among workers in health care settings? Implementation Science 2011; 6: 47.

10. Song JY, et al. Effect of a hospital campaign for influenza vaccination of healthcare workers. Infection Control and Hospital Epidemiology 2006; 27: 612-617. 